\title{
Magnetic Wave Propagation in a Periodic Medium
}

CHARLES ELACHI, MEMBER, IEEE

Abstract-The propagation of magnetic waves in an infinite medium with a periodic dielectric constant is studied as a simplified example to evaluate the applications of periodic structures. Specifically, the use of those structures for filtering and distributed feedback is investigated, and a new scheme for the generation of magnetic waves using drifting charges and a distributed feedback configuration similar to DFB lasers is studied in some detail.

\section{INTRODUCTION.}

Magnetic materials having permanent microscopic moments (ferromagnets, ferrimagnets, and antiferromagnets) can support highly dispersive magnetic waves with phase velocities which span the range from electromagnetic to elastic waves [1]. These waves are used in a number of applications ranging from delay lines and pulse compression filters to frequency translation and time inversion and scaling [see 1 for review]. In this paper we consider the propagation of these waves in a periodic structure. For simplicity we will consider the case of plane waves in a linear isotropic medium with a periodic dielectric constant. Adequate periodicity would lead to Bragg selective reflection and therefore filtering. However, if the medium is amplifying due to the presence of drifting charges [2], then the Bragg reflection would play the role of distributed feedback (DFB) in an amplifier, thus leading to oscillation. This concept has recently been introduced to the field of lasers [3]-[7] and acoustic microwave generation [8]. The use of DFB to develop magnetic wave generators is discussed in detail.

\section{FORMULATION ANDD SOLUTION}

Let us consider a magnetic medium characterized by a permanent magnetization $\boldsymbol{M}_{s}$, a dc magnetic field $\boldsymbol{H}$, a space dependent dielectric constant $\epsilon(z)$, and a magnetic permeability $\mu$. The two vectors $M_{S}$ and $\boldsymbol{H}$ are supposed to be parallel to the $z$ axis (Figure 1 ), which is also the wave propagation axis. The magnetization $(m)$ and electromagnetic $(e, b)$ rf fields in such a medium are related by $[1$ with units change] :

$$
\begin{aligned}
\frac{\partial m}{\partial t} & =-\gamma j_{z} \times\left[M_{s} b-\left(H-D \nabla^{2}\right) m\right] \\
\nabla \times b & =\epsilon(z) \frac{\partial e}{\partial t}
\end{aligned}
$$

Manuscript received March 11, 1974; revised Ocrober 15, 1974. This paper represents the results of one phase of research carried out at the Jet Propulsion Laboratory, California Institute of Technology, under Contract NAS7-100 sponsored by the National Aeronautics and Space Administration.

The author is with Jet Propulsion Laboratory, California Institute of Technology, Pasadena, Calif. 91103.

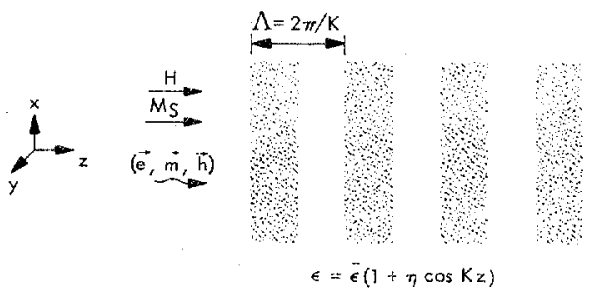

Fig. 1. Geometry studied. $H$ and $M_{S}$ are the dc fields. $e, m$, and $h$ are the ac fields.

$$
\nabla \times e=-\mu\left(\frac{\partial b}{\partial t}+\frac{\partial m}{\partial t}\right)
$$

where $D$ is the exchange constant, $\gamma$ is the gyromagnetic ratio, and $j_{z}$ is the unit vector along the $z$-axis. These formulas assume that $H$ and $M_{s}$ are independent of spatial coordinates and that the anisotropy field is negligible (which is the case in the most commonly used magnetoelastic medium, Yttrium Iron Garnet). Equation 1 implies that $m$ has only transverse components.

Let us assume the case where the dielectric constant inhomogeneity is periodic of the type:

$$
\epsilon(z)=\bar{\epsilon}(1+\eta \cos K z)=\bar{\epsilon}\left(1+\frac{\eta}{2} e^{i K z}+\frac{\eta}{2} e^{-i K z}\right)
$$

where $\eta<<1$ is a modulation factor, $K=2 \pi / \Lambda$, and $\Lambda$ is the inhomogeneity period. The solution for the above system of equations can be written in a Floquet form [9], [10] which consists of an infinite number of space-harmonics:

$$
(\boldsymbol{m}, \boldsymbol{e}, \boldsymbol{b})=e^{-i \omega t} \sum_{n=-\infty}^{n=+\infty}\left(\boldsymbol{m}_{n}, \boldsymbol{e}_{n}, b_{n}\right) \exp \left(i \beta_{n} z\right)
$$

where $\beta_{n}=\beta+n K$. The index $n$ corresponds to the different space harmonics, and $\beta$ is an unknown wavevector which will be related to the frequency $\omega / 2 \pi$ by the dispersion equation. Replacing each term in (1)-(3) by its Floquet form, and solving for the terms of identical wavevectors, we find the following systems of infinite equations which relate the different harmonics of the electric field:

$$
\begin{array}{r}
\left(k^{2}-\beta_{n}^{2}\right) j_{z} \times e_{n}+i \frac{\gamma}{\omega}\left\{\left(k^{2}-\beta_{n}^{2}\right)\left(H+D \beta_{n}^{2}\right)+k^{2} M_{s}\right\} e_{n} \\
+\frac{\eta}{2} k^{2}\left[i \frac{\gamma}{\omega}\left(H+M_{s}+D \beta_{n}^{2}\right)+j_{z} \times\right]\left(e_{n+1}+e_{n-1}\right)=0
\end{array}
$$

where $k^{2}=\mu \bar{\epsilon} \omega^{2}$. This system car be simplified if we split the field in its positive and negative circular components (i.e., $e^{+}=$ 


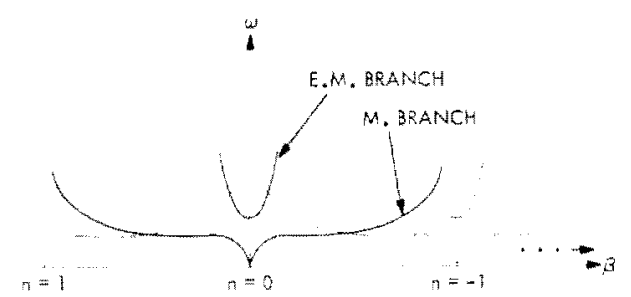

Fig. 2. Brillouin diagram for a periodic magnetic medium. The heavy curves correspond to the homogeneous case. All other subdiagrams correspond to higher order space harmonics. The scales arc adjusted for clarity.

$$
\begin{gathered}
\left.e_{x}+i e_{y} \text { and } e^{-n}=e_{x}-i e_{y}\right), \text { leading to: } \\
d_{n}^{+} e_{n}^{+}+e_{n+1}^{+}+e_{n-1}^{+}=0 \\
d_{n}^{-} e_{n}^{-}+e_{n+1}^{-}+e_{n-1}^{-}=0
\end{gathered}
$$

where:

$$
d_{n}^{ \pm}=\frac{2}{\eta}\left[1-\frac{\beta_{n}^{2}}{k^{2}}\left(1-\frac{ \pm \gamma M_{s}}{\omega \pm \gamma\left(M_{s}+H+D \beta_{n}^{2}\right)}\right)\right] .
$$

This shows that the coupling occurs between the spaceharmonics of identical circular rotation. The $n$th harmonic is directly coupled to the $n-1$ st and $n+1$ st harmonics, and then indirectly to the others. This is due to the cosine nature of $\epsilon(z)$. For the case of general periodicity, direct coupling could occur between any two space-harmonics. The dispersion equation is obtained from the nontriviality condition:

$$
\text { determinant of (7) or (8) equals zero. }
$$

If we take the limit $\eta=0$, then (7) and (8) reduce to $\eta d_{n}=0$ which gives:

$$
\left[\omega \pm \gamma\left(H+D \beta_{n}^{2}\right)\right]\left(1-\frac{\beta_{n}^{2}}{k^{2}}\right) \pm \gamma M_{s}=0
$$

which is the well known dispersion equation in a homogeneous magnetic medium [1].

\section{BRILLOUIN DIAGRAM AND COUPLING REGIONS}

The Brillouin diagram which corresponds to (11) consists of an infinite number of "subdiagrams" each identical to the homogeneous case diagram (Figure 2). Each subdiagram corresponds to a space-harmonic $n$. For $\eta=0$, only the spaceharmonic $n=0$ has a physical meaning.

For $\eta \neq 0$, strong phase matched coupling occurs between different space harmonics at their intersection point. The detailed study of these coupling regions would require a numerical solution of $(10)$. However, much insight can be obtained by studying the limit $\eta$ small using a first order Taylor series expansion.

Without loss of generality, let us consider the region close to the intersection point $(\omega, \beta)$ of the $n=0$ and $n=-1$ space harmonics. Neglecting higher harmonics, we get the dispersion relation:

$$
d_{0} d_{-1}=1 \text {. }
$$

The frequency and wavevector can be written as $\omega+\Delta \omega$ and $\beta+\Delta \beta$. From (9) and (12) we could express $\Delta \omega$ as a function of $\Delta \beta$.

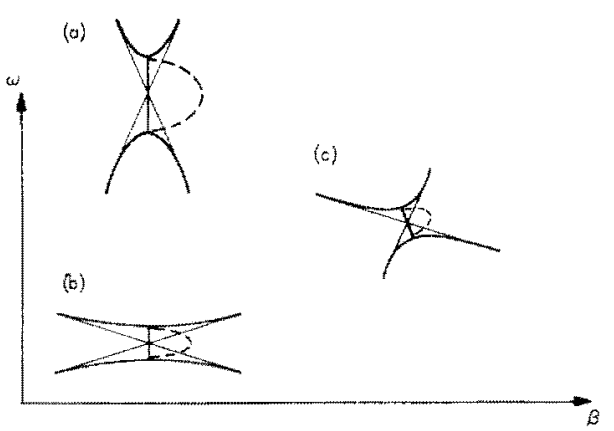

Fig. 3. Different types of contradirectional interations. The light lines correspond to $\eta=0$ (no perturbation). The heavy line is $R e(\beta)$ and the dashed line is Im ( $\beta$ ). (a) corresponds to e,m, - e.m. coupling, (b) corresponds to $m,-m$. coupling, (c) corresponds to e.m. $-m$. coupling.

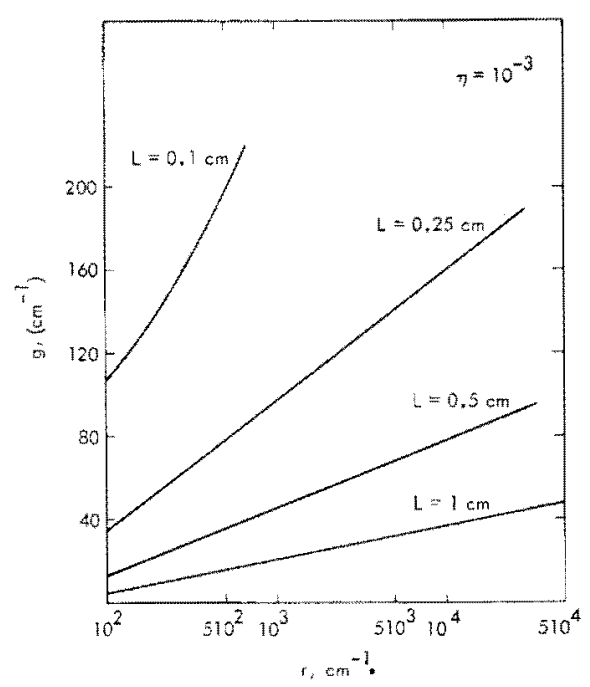

Fig. 4. Threshold gain coefficient g required for self sustained oscilla tion as a function of the wavevector $r$ for different values of $L$.

As we are interested in the coupling between spaceharmonics, we stay outside the "natural" coupling region between the magnetic and electromagnetic waves.

Three types of backward coupling are possible (Figure 3):

1. Electromagnetic-Electromagnetic coupling (Figure 3a):

$\beta=k \Rightarrow K=2 k$

and

$(\Delta \omega / \omega)^{2}-(\Delta \beta / \beta)^{2}=(\eta / 4)^{2}$.

2. Magnetic-Magnetic coupling (Figure 3b):

$\beta=\left(\frac{\omega-\gamma H}{\gamma D}\right)^{1 / 2} \Rightarrow K=2\left(\frac{\omega-\gamma H}{\gamma D}\right)^{1 / 2}$

and

$$
(1-a)^{2}(\Delta \omega / \omega)^{2}-(1+b)^{2}(\Delta \beta / \beta)^{2}=(\eta / 4)^{2} .
$$

3. Magnetic-Electromagnetic coupling (Figure 3c):

$$
\beta=k=K-\left(\frac{\omega-\gamma H}{\gamma D}\right)^{1 / 2}
$$

and

$$
(\Delta \omega / \omega-\Delta \beta / \beta)[(1-a) \Delta \omega / \omega+(1+b) \Delta \beta / \beta]=(\eta / 4)^{2}
$$




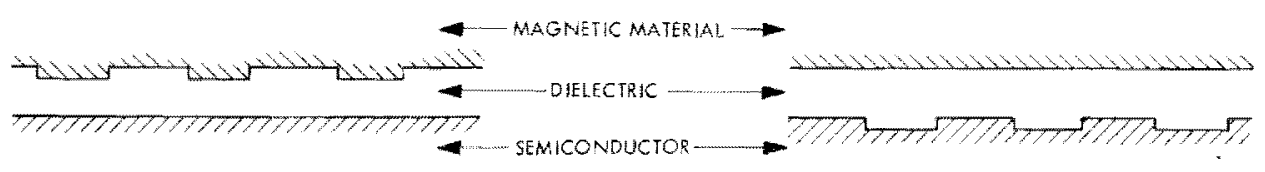

Fig. 5. Some practical configurations for a DFB magnetic wave oscillator.

where:

$$
\begin{aligned}
a & =\frac{\omega}{2 \gamma M_{s}}\left(\frac{r^{2}-k^{2}}{r k}\right)^{2} \\
b & =\gamma D a r^{2} / 2 \omega \\
r^{2} & =(\omega-\gamma H) / \gamma D .
\end{aligned}
$$

In these equations, we remark that near $\Delta \omega=0$ (phase matching region), the solution for $\Delta \beta$ is imaginary (i.e., $\beta$ is complex). This corresponds to a stop band coupling where one wave loses energy to the other one which is propagating in the opposite direction. The coupling region is characterized by two parameters (Figure 3): the relative bandwidth $\Omega / \omega$ (region of complex $\beta$ ) and the coupling coefficient $\chi$ (imaginary $\beta$ for $\Delta \omega=0$ ). In the case of magnetic-magnetic coupling we get from (16):

$$
\begin{aligned}
\Omega / \omega & =\eta / 4(1-a) \\
\chi & =\eta r / 4(1+b) .
\end{aligned}
$$

The parameter $\chi$ represents the strength of the distributed feedback. If the magnetic medium is passive, then $\chi$ expresses the filtering efficiency of the periodic structure. If the medium is active (due to the presence of drifting charges), then $\chi$ corresponds to the strength of the feedback which would allow the system to oscillate as discussed in the following section.

\section{DFB MAGNETIC WAVE GENERATOR}

Kogelnik and Shank [3] have conducted a detailed study of the DFB concept in the case where both the forward and backward wave are equally amplified. Elachi et al. [7], [11] have generalized their study to the case where the two waves are not equally amplified as is the case here. The threshold condition which relates the coupling coefficient $X$, the average gain coefficient $\bar{g}$, and the length of the coupling region $L$ is:

$$
\chi= \pm i \psi / \sinh \psi L
$$

where

$$
\begin{aligned}
& \psi=\left[\chi^{2}+\left(\bar{g}-i \beta \frac{1-a}{1+b} \frac{\Delta \omega}{\omega}\right)^{2}\right]^{1 / 2} \\
& \bar{g}=g / 2 \\
& g=\text { magnetic wave gain coefficient. }
\end{aligned}
$$

In the limit of high gain (i.e., $g>>$ ) which is usually the case here (see later), the above equation gives $[3]$ :

$$
g L e^{-g L / 2}=\chi L \text {. }
$$

The magnetic wave gain was derived by many authors [1], [12], [13], and their results will be used here to evaluate the feasibility of a spin wave oscillator. The periodicity required for oscillation (i.e., phase matched feedback) at $\omega$ is given by (from (15)):

$$
\Lambda=2 \pi / K=\pi[\gamma D /(\omega-\gamma H)]^{1 / 2} .
$$

The solution of (21) is multivalued [3]; however, we will only consider the first solution which is the closest to the exact Bragg condition (i.e., $\Delta \omega=0$ ).

In the case of pure magnetic wave, $r>k$. Thus the coupling coefficient $\chi$ reduces to:

$$
\chi=\eta M_{s} / 4 D r^{3}
$$

which is usually $<<g$ and therefore the threshold relation is (22).

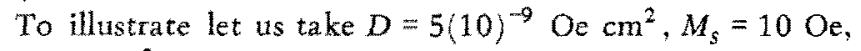
and $\eta=10^{-3}$. In Figure 4 we plotted the gain coefficient $g$ required for oscillation as a function of $r$ and for different values of $L$. We see that for a $0.5 \mathrm{~cm}$ long device, a gain coefficient of $100 \mathrm{~cm}^{-1}$ is enough to have oscillation at wavenumbers up to $5(10)^{4} \mathrm{~cm}^{-1}$ (i.e., down to wavelength of $\simeq 6 \mu \mathrm{m}$ ). For a $1 \mathrm{~cm}$ long device, a gain coefficient of $50 \mathrm{~cm}^{-1}$ would allow oscillations down to about $1 \mu \mathrm{m}$ wavelength. These gain coefficients are possible in some ferromagnetic semiconductors. In fact, Vural [13] has estimated gains per centimeter of $10^{5}$ in an interpenetrating system of semiconductor ( $\mathrm{Si}, \mathrm{Ge}, \mathrm{InSb}$ ) and YIG. Considering the curves derived by Steele and Vural [12], we can see that gains of many $100 \mathrm{~cm}^{-1}$ can be achieved in the $X$-band region even with magnetic and electric losses. However, in pracrice, the most feasible configuration for a DFB oscillator might be similar to Schloeman's [14] amplifier which consists of neighboring semiconductor and ferromagnetic medium separated by a dielectric (Figure 5). It should be mentioned that the feedback does not specifically require periodicity in the dielectric constant. In fact, surface corrugation or the periodicity of any parameter which has an effect on the propagation of the wave, could be satisfactory (Figure 5). Similarly, the periodicity does not have to be sinusoidal.

\section{CONCLUSION}

We conclude that the distributed feedback concept is promising enough to be seriously considered for the development of magnetic wave oscillators. The shortest oscillation wavelength will be limited by the shorted periodicity $\Lambda$ feasible. Presently, holographic techniques are used to generate surface corrugations with a period of $0.1 \mu \mathrm{m}$ [15] which will be more than satisfactory.

\section{REFERENCES}

[1] B. A. Auld, Applied Solid State Science, volume 2, Edited by R. Wolfe, Academic Press, N.Y. (1971).

(2) H. Kogelnik and C. V. Shank, J. Appl. Phys, 43, 2327 (1972).

[3] H. Kogelnik and C. V. Shank, Appl Phys Lett., 18, 152(1971).

4) K. O. Hill and A. Watanabe, Opt. Comm., 5, 389 (1972),

5. H. Nakamura, A. Yariv, H. W. Yen, S. Somekh, and H. L. Garvin, Appl. Phys. Lett., 22, 515 (1973). 
[6] C. Elachi, G. Evans, and F. Grunthaner, Applied Optics, Jan. 1975.

[7] C. Elachi, G. Evans and C. Yeh, IEEE Trans. on MTT, to be published.

[8] C. Elachi, Applied Physics, to be published.

[9] T. Tamir, H. C. Wang, and A. A. Oliner, IEEE Trans, MTT-12, $323,(1964)$.

[10] C. Elachi and C. Yeh, J. Appl. Phys., 414, 3146 (1973).
[11] C. Elachi, G. Evans and C. Yeh, Integrated Optics Conference, New Orleans, 1974

[12] H. C. Steele and B. Vural, Wave Interactions in solid state plasmas, McGraw-Hill, N.Y. (1969).

[13] B. Vural, J. Appl. Phys., 37, 1030 (1966).

14] E. Schloemann, J. Appl. Phys., 40,1622 (1964).

[15] C. V. Shank and R. V. Schmidt, Appl. Phys. Lett., 23, 156 (1973).

\title{
Calculation and Optimization of the Magnets for an Electromagnetic Levitation System
}

\author{
PETER APPUN AND GERD R. RITTER
}

\begin{abstract}
The calculation of the static behavior of the magnets of an electromagnetic levitation system can be performed using a numerical field calculation method. The agreement of measured with calculated values is satisfactory. Besides the excitation losses of the magnets, there are also losses due to eddy currents in the rails. Some measurements, calculations, and important parameters for these eddy current effects are also discussed.

Not only the analysis of the magnets but also the design, optimized with regard to certain conditions is important. Some examples show the optimization of the magnet using a nonlinear optimization method.

The paper closes with the description of the dynamic behavior of the magnet using different linear and nonlinear models.
\end{abstract}

\section{INTRODUCTION}

For high-speed ground transportation systems using fixed guideways it may be economically justifiable to not only have a noncontact propulsion system but also to have a noncontact means of suspension. The magnetic and air-cushion levitation schemes are the two main competitors in this field today. One differentiates in the magnetic levitation schemes between electrodynamic and electromagnetic methods, the latter being, in part, discussed here.

The magnets themselves are an essential component of an electromagnetic levitation sy stem for a guided-rail vehicle. Their weight represents a considerable proportion of the total vehicle weight. The cross-section and the cost of the rails which are set alongside the guideway are chiefly determined by the design of the magnets. Further equipment which requires space in the

Manuscript received July 10, 1974; revised September 6, 1974. This work was supported by the Bundesministerium für Forschung und Technologie of the Federal Republic of Germany. A summary of this report was presented at the INTERMAG Conference, Toronto, Canada, May 1974.

P. Appun is with Brown, Boveri \& Cie. AG, Mannheim, Germany.

G. R. Ritter was with Brown, Boveri \& Cie. AG, Mannheim, Germany, He is now with the German Federal Railway Board. vehicle and causes additional weight is necessary for the magnet power supply. Because of the braking force caused by the eddy currents a higher propulsion force is required.

Besides the above mentioned design problems which refer to steady state conditions, the second most important problem area concerns dynamic operations because an electromagnetic levitation system is unstable with regard to air-gap variations. The recognition of the dynamic behavior of the magnets is therefore important for the development of the necessary position control.

\section{FIELD AND FORCE CALCULATIONS}

An initial condition for the investigation of an electromagnetic levitation system is the availability of a sufficiently exact calculation-method for the static behavior of the magnets. A program has been developed by $B B C$ which enables numerical calculations on the magnetic field, force, and inductances to be carried out. Various magnet and rail geometries; i.e., magnets with $\mathrm{U}$ - and E-profiles and various winding arrangements with flat and U-profile rails can be treated.

The calculation method used was a numerical one using finite differences, the principles of which are well known; e.g., $[1,2]$. Magnetic fields of arrangements with exciting currents, air sections, and iron sections with nonlinear magnetization characteristics, can be successfully numerically calculated.

The necessary calculation steps are

the selection of the vector-potential as the field-dimension, the division of the arrangement through a grid system into finite elements, as shown in Fig. 1,

the correlation of definite material characteristics for each element,

the specification of the difference equation system for the vector-potentials at the various grid points,

the solution of the equation system using elimination and iterative methods which take into consideration the nonlinear magnetic characteristic of the iron, which can be given in the form of the characteristic curve, 